\title{
Design of a Novel Micro-Laser Formed by Monolithic Integration of a III-V Pillar With a Silicon Photonic Crystal Cavity
}

\author{
Zhechao Wang, Member, IEEE, Bin Tian, and Dries Van Thourhout, Member, IEEE
}

\begin{abstract}
A novel micro-laser configuration formed by integrating an InGaAs/InP pillar with a silicon photonic crystal cavity is proposed and analyzed in detail. Special attention is paid to designing the cavity such that it can accommodate large-size pillars without performance compromise. The Purcell effect is studied and predicted to be significant because of the close interaction between the cavity modes and the gain medium. An overall quality factor as high as $1 \times 10^{5}$ and a spontaneous emission factor close to unity are predicted. Possible limiting factors for laser performance, such as surface non-radiative recombination and the thermal dissipation properties are analyzed, and it is found that the proposed laser design is very robust. This comprehensive analysis suggests that the proposed micro-laser is a promising candidate for large-scale integration of micro-lasers on silicon for low power consumption applications, such as intra-chip optical communications.
\end{abstract}

Index Terms-Laser materials, micro-resonator, photonic crystal laser, Purcell effect, silicon photonics.

\section{INTRODUCTION}

D RIVEN by demands from optical I/O, telecommunications, optical computing and other applications, extensive research is being carried out in the field of integrating III-Vs on silicon [1]-[7]. Besides bonding based integration methods [1], [2], which have been very successful in demonstrating a wide range of functional diversity and good device performance, epitaxial growth of III-Vs on silicon recently regained interest both for material science and optical engineering [3]-[5]. Despite the large lattice mismatch between silicon and typical III-V materials, considerable improvements in both material quality and device performance were achieved through technologies such as lattice-matched growth [6], [7], the use of a self-organized dislocation network [8], [9], lateral epitaxial overgrowth [10] or quantum dot epitaxy [11], [12]. All of these technologies typically are used for growing large areas of III-V based materials. While this is interesting for some applications, this is

Manuscript received December 12, 2012; revised February 07, 2013; accepted March 10, 2013. Date of publication March 13, 2013; date of current version March 29, 2013. This work was supported in part by the EU ERC starting grant ULPPIC and the Belgian Science Policy Office (IAP P7/35-photonics@be).

The authors are with the Photonics Research Group, INTEC-department, Ghent University-IMEC, Ghent 9000, Belgium and Center for Nano- and Biophotonics (NB-Photonics), Ghent University, Ghent 9000, Belgium (e-mail: zhechao.wang@intec.ugent.be; bin.tian@intec.ugent.be; dries.vanthourhout@intec.ugent.be).

Color versions of one or more of the figures in this paper are available online at http://ieeexplore.ieee.org.

Digital Object Identifier 10.1109/JLT.2013.2252600 not always strictly necessary for the device under consideration. In such cases it might be more interesting to focus on a localized epitaxial growth process, which consumes much less of the expensive growth precursors and might require less or even no processing steps after epitaxy. One approach for localized growing good quality material is using the so-called defect necking effect [13]. When growing in a narrow trench, this effect prevents dislocations to propagate upwards from the lattice mismatched interface. Using this approach Wang et al. [14], [15] demonstrated the growth of defect-free InP in narrow silica trenches formed on a silicon substrate using MOCVD. While originally targeted towards realizing high-performance transistors, these pillars possibly may also form the basis for realizing low threshold micro-sources on silicon.

The aim of this paper is to investigate through detailed theoretical and numerical analysis if the combination of such a $\mathrm{InP} / \mathrm{InGaAsP}$ pillar with a predefined silicon photonic crystal $(\mathrm{PhC})$ cavity can form a low threshold laser. The $\mathrm{PhC}$ cavity is employed here mainly for its small mode volume $(V)$ and high quality factor $(Q)$, which both should help to minimize the threshold current [16]-[18]. In addition, the associated Purcell effect [19] could further reduce the threshold current [20] and increase the modulation bandwidth [21], [22]. Threshold-less lasing has been demonstrated in devices where the spontaneous emission factor $(\beta)$ was increased to unity [23]-[26]. In this work we will focus on one-dimensional (1-D) PhC cavities, which, compared to the more extensively explored two-dimensional $\mathrm{PhC}$ cavities, can achieve a sufficiently high $\mathrm{Q} / \mathrm{V}$ factor within a much smaller overall footprint. Although the introduction of a high-index pillar within the cavity degrades its performance [27], we will show that, in the proposed case, the cavity performance can be almost completely recovered by engineering the pillar geometry.

\section{Laser Configuration, Gain Threshold and Purcell EFFECT}

Fig. 1 shows the proposed laser configuration, in which a cavity is formed by etching two one-dimensional hole-arrays into a silicon waveguide with a thickness of $220 \mathrm{~nm}$. Optical gain is provided by an $\mathrm{InP} / \mathrm{InGaAs} / \mathrm{InP}$ heterostructure, located in the center of the cavity. $h_{\text {pillar }}$ indicates the total height of the III-V pillar.

Very similar to the transistor structure proposed in reference [14], [15], this pillar can be fabricated by etching a trench through the silicon waveguide and silica buffer, and then performing epitaxial growth of III-Vs directly on the silicon 


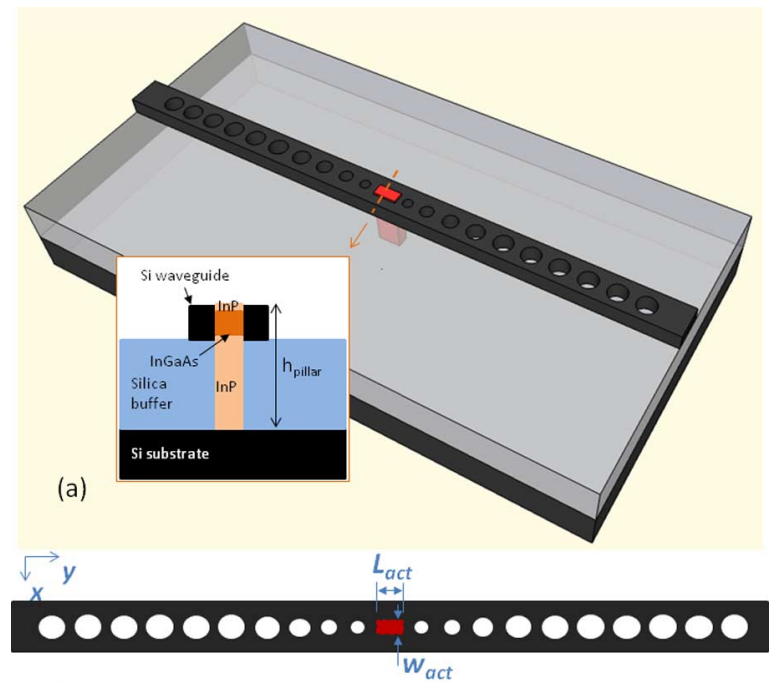

(b)

Fig. 1. (a) Three-dimensional sketch and (b) top view of the 1-D PhC cavity coupled with an InGaAs/InP pillar grown from the silicon substrate underneath. Insert: cross section view of the central cavity region. The InGaAs layer is 150 $\mathrm{nm}$ thick.

substrate. An insert is provided to give the cross section view of the heterostructure inside the trench. The InGaAs layer, which provides the optical gain, is sandwiched between two InP layers. It is assumed to be $150 \mathrm{~nm}$ thick and is aligned in the middle of the silicon waveguide for better optical confinement.

Different from a conventional waveguide based laser, the $\mathrm{PhC}$ cavity in the proposed laser configuration tightly confines the optical mode into a very small region $\left(\sim(\lambda / 2 n)^{3}\right)$. The strong coupling between optical modes and gain medium can change the spontaneous emission rate dramatically. This so-called Purcell effect [19] can be quantified through the Purcell factor $F$, whose expression is given by [24]-[26]:

$$
F=\frac{3}{4 \pi^{2}} \frac{\lambda}{\Delta \lambda} \frac{\left(\frac{\lambda_{c}}{n}\right)^{3}}{V_{\text {eff }}},
$$

where $V_{\text {eff }}$, and $\lambda_{c}$ are the effective mode volume, and central wavelength of the cavity mode. $\mathrm{n}$ is the refractive index at the field maximum. $\Delta \lambda$ is the larger of either the electron transition broadening or the cavity resonance linewidth. Given the high quality factor of the $\mathrm{PhC}$ cavity, homogeneous broadening of the electron transition becomes dominant ( $\sim \mathrm{nm}$ [29]) here and determines $\Delta \lambda$. The expression for the mode volume $V_{\text {eff }}$ is given by:

$$
V_{\mathrm{eff}}=\int v \frac{n(r)^{2}|E(r)|^{2}}{\max \left(n(r)^{2}|E(r)|^{2}\right)} d r^{3},
$$

where $E$ is the electric field, $n(r)$ is the spatially dependent refractive index distribution and $r$ is the spatial coordinate.

Thanks to the Purcell effect, the spontaneous emission factor $\beta$, being the fraction of spontaneous emission radiated into the resonance mode can be greatly enhanced and can be calculated as:

$$
\beta=\frac{F}{F+\alpha},
$$

$\alpha$ thereby represents the fraction of spontaneous emission that is coupled into leaky modes and all nonlasing modes. The coupling to nonlasing modes can be greatly suppressed in a nanocavity that has a very large free spectral range, while the coupling to leaky modes has been calculated to be close to unit [26] for a structure that is very similar to the one discussed in this paper. For simplicity, $a \approx 1$ is used in the following calculations.

Taking the Purcell effect into consideration, the rate equations of an optically pumped laser can be rewritten as:

$$
\begin{aligned}
\frac{d N}{d t}= & -v_{g} S G(N)-\left(\frac{A_{a}}{V_{a}} v_{s} N+F B N^{2}+C N^{3}\right) \\
& +\frac{P_{a b s}}{V_{a} E_{p}} \\
\frac{d S}{d t}= & \Gamma v_{g} S G(N)+\Gamma \beta F B N^{2}-\frac{S}{\tau_{p}},
\end{aligned}
$$

where $N$ and $S$ are the carrier and photon density, $A_{a}$ is the active region surface area, $v_{g}$ is the group velocity, $v_{s}$ is the surface recombination velocity, $B$ is the spontaneous emission rate without cavity confinement, $C$ is the Auger recombination rate, $P_{\mathrm{abs}}$ is the absorbed pump power, $E_{p}$ is the emitted photon energy and $\tau_{p}$ is the photon lifetime. $G$ is the $N$ dependent material gain, which can be expressed as $G(N)=$ $g_{0} \ln \left(\left(N+N_{s}\right) /\left(N_{\mathrm{tr}}+N_{s}\right)\right)$ [30]. $g_{0}$ is the differential gain coefficient, $N_{\mathrm{tr}}$ is the transparent carrier density and $N_{s}$ is a fitting parameter. The optical gain calculated at threshold is used as a measure to evaluate the overall laser cavity performance. However, due to the large contribution of spontaneous emission in a micro-cavity with high $\beta$. it is not straightforward to give a simple expression for the gain threshold [28]. It is also tricky to define the threshold of such laser cavities because of the smooth transition from spontaneous emission dominated operation towards laser operation. In Sections III-VI, we will adopt the threshold condition given in [28] and defined as the operating point whereby the net simulated emission equals the spontaneous emission. Full simulation based on the rate equations with carrier-dependent gain was performed to find out the carrier density at threshold, based on which the threshold gain is calculated.

\section{One Dimensional Photonic Crystal Cavity Design}

In order to achieve low threshold laser operation, the design of a cavity with high quality factor is critical. The one dimensional (1-D) PhC cavity first proposed by Foresi in 1997 [16] was still relatively lossy $\left(Q<10^{3}\right)$. Recent work however has demonstrated Q-factors up to $10^{6}$ for such cavities [31], [32]. Here, we adopt an optimization approach similar to that used in the latter case: gradually tapering hole diameter and $\mathrm{PhC}$ period to realize a smooth transition between waveguide mode and Bloch mode. In this way, the cavity loss can be effectively reduced. Three dimensional (3-D) finite-difference time-domain (FDTD) modeling was used as the simulation tool, and the silicon waveguide dimension was set to $520 \mathrm{~nm} \times 220 \mathrm{~nm}$ in all simulations. 


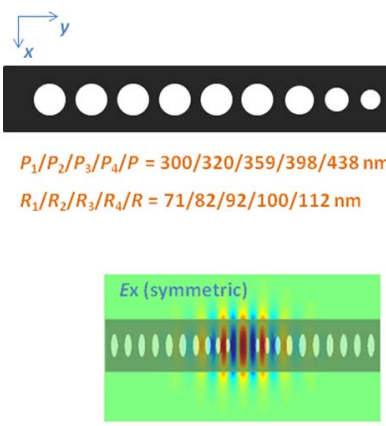

(b)
$L_{\text {cav }} P_{1} P_{1}^{P_{2}} P_{3} P_{4} P_{4}$ 000000000

Tapered hole array Uniform PhC

(a)

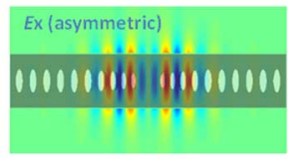

(c)

Fig. 2. (a) Top view of the optimized $\mathrm{PhC}$ cavity, which has two typical mode distributions: (b) symmetric mode and (c) asymmetric mode. The PhC cavity outline has also been plotted for better visualization of the mode confinement. The PhC cavity optimized parameters are listed in Fig. 2(a).

A sketch of the $\mathrm{PhC}$ cavity with optimal geometrical parameters can be found in Fig. 2(a). Six holes with uniform period $(P)$ and radius $(R)$ are employed as the main $\mathrm{PhC}$ reflector, while four holes with varying periods $\left(P_{1}, P_{2}, P_{3}, P_{4}\right)$ and radius $\left(R_{1}, R_{2}, R_{3}, R_{4}\right)$ are placed between the $\mathrm{PhC}$ mirror and the cavity region. The cavity length $\left(L_{\text {cav }}\right)$ is defined as the distance between the two inner period sections. The optimal design can be found in the insert of Fig. 2(a). Since the supported quasi-transverse-magnetic (quasi-TM) modes exhibit a high loss and are located far away from the maximum of the material gain spectrum, we will only consider quasi-TE modes in the following.

Generally speaking, there are two different types of modes that are supported by the cavity: symmetric modes and asymmetric modes. Their typical mode distributions $\left(E_{x}\right.$ field) can be found in Fig. 2(b) and (c). Asymmetric modes have an amplitude minimum at the cavity center. Introduction of a III-V pillar (see the dashed rectangle in Fig. 2(a)) will severely disturb the mode distribution, leading to higher cavity loss. To the contrary, the mode distribution of symmetric modes will not change much by the III-V pillar, leading to low cavity loss. The large quality factor difference between symmetric and asymmetric modes will allow single mode operation. Therefore, we will only consider symmetric modes in the following discussion.

Fig. 3 shows the calculated resonance wavelengths of the first two symmetric modes against the cavity length. For shorter cavity lengths, the first order mode is located at the band edge. Since its electric field is weakly confined, it will leak through the finite length $\mathrm{PhC}$ (see a typical mode distribution in the insert of Fig. 3) and one can expect low quality factors for the first order modes. At the same time, when the cavity length is relatively long ( $>600 \mathrm{~nm}$ ), second order modes are well located in the bandgap center, thus high quality factors, i.e., low cavity loss, can be expected. A longer cavity length is also preferable from the perspective of the envisaged laser application, since in that case larger III-V pillars can be accommodated by the cavity. Therefore, higher optical confinement in the active region can be achieved, providing more optical gain. In the following discussion, we will adopt $630 \mathrm{~nm}$ as the optimal cavity length. The corresponding quality factor-without III-V pillar-is calculated to be $5 \times 10^{5}$.

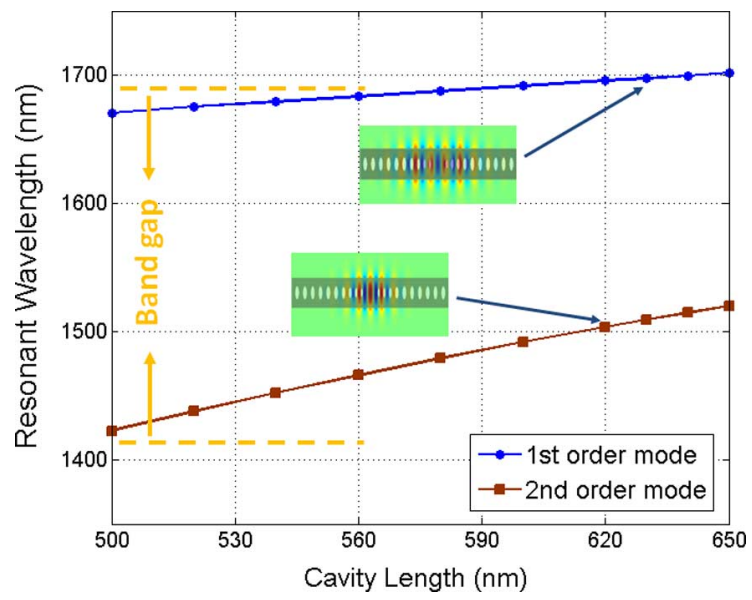

Fig. 3. Resonant wavelengths of the first and second order symmetric modes for different cavity lengths. Insert: typical optical mode distributions of thee first and second order modes. Dashed lines indicate the bandgap of the 1D-photonic crystal forming the mirrors.

\section{III-V Pillar Coupled With the PhC CAVITy}

By introducing InGaAs/InP pillars inside the $\mathrm{PhC}$ cavity, the optical field can be amplified by pumping the III-V heterostructure. When the optical gain overcomes the cavity loss, laser behavior is expected. In this section, we present detailed simulation results of the cavity. As a starting point, we calculate the quality factor of a PhC cavity with a $150 \mathrm{~nm} \times 200 \mathrm{~nm}$ sized InGaAs/InP pillar. The total pillar height (see Fig. 1) is assumed to be $1 \mu \mathrm{m}$ in the following analysis (the corresponding $\mathrm{SiO}_{2}$ thickness is $780 \mathrm{~nm}$ ). This is a good comprise between cavity performance and fabrication challenges, as will be discussed later. The quality factor is calculated to be $2.5 \times 10^{3}$, which is two orders of magnitude lower than the cavity without a pillar. The extra cavity loss could come from the enhanced upwards out-of-plane light leakage due to the disturbance of the optimal mode distribution, and/or due to the downwards light leakage through the high index pillar that physically connects the cavity and substrate. The out-of-plane leakage can be investigated by decomposing the electric field into a set of plane wave components with various k-vectors using a spatial Fourier transformation and analyze the fraction of $\mathrm{k}$-vector that falls into the light cone [33]. Fig. 4(a) and (b) shows 2-D Fourier transforms of the cavity modes without and with a pillar. The white circle indicates the leakage region. As one can see, the k-vector distribution in the white circle is barely changed after introducing the pillar, which indicates that the out-of-plane leakage contributes little to the enhanced cavity loss.

The downwards leakage can be visualized by plotting the cross section of the cavity mode in natural log scale (see Fig. 4(c)). Due to the high-index nature of the InGaAs/InP pillar, the optical mode is guided towards the substrate, leading to leakage loss. Luckily, the reduced quality factor can be partially restored by optimizing the InGaAs/InP pillar geometry. Fig. 4(e) shows the quality factor calculated for different pillar lengths. When $L_{\text {act }}$ is larger than $450 \mathrm{~nm}$, the quality factor rapidly increases by one order of magnitude, and then saturates when $L_{\text {act }}$ approaches the physical limit of the cavity length. Fig. 4(d) shows the cross section of the cavity mode when 

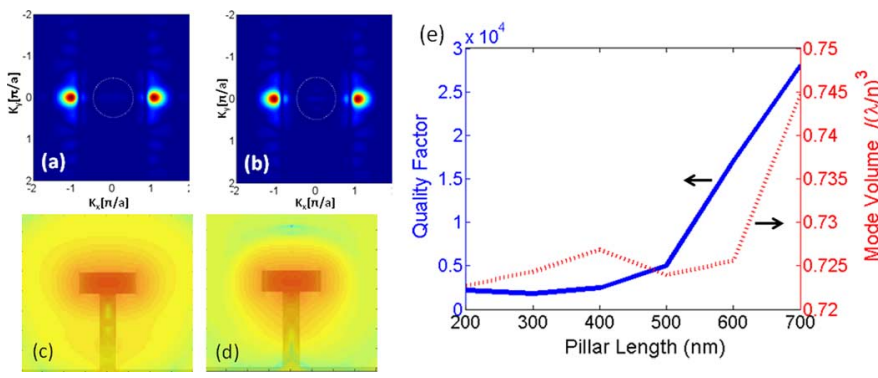

Fig. 4. 2D Fourier transforms of cavity modes (a) without and (b) with a In$\mathrm{GaAs} / \mathrm{InP}$ pillar. Cross-sectional view of the field distributions of cavity modes with a (c) $200 \mathrm{~nm}$ and (d) $700 \mathrm{~nm}$ long InGaAs/InP pillar, on a logarithmic scale. (e) calculated cavity quality factors (solid line) and mode volumes (dashed line) for different pillar sizes.

$L_{\text {act }}=700 \mathrm{~nm}$. The increased volume of InGaAs material $\left(n_{\text {InGaAs }}=3.72\right)$ helps to lift up the mode profile from the substrate, which reduces the leakage considerably. Simulation shows that the leakage loss is almost one order of magnitude lower when $L_{\text {act }}=700 \mathrm{~nm}$, compared to the case when $L_{\text {act }}=200 \mathrm{~nm}$. We also plot the mode volume against pillar size in Fig. 4(e) by using expression (3), and one can find that the mode volume increases as pillar size increases, although the absolute value change is very limited.

A more comprehensive analysis of the laser mode is presented in Fig. 5, in which quality factor, resonant wavelength, confinement factor, threshold gain and Purcell factor are scanned for different pillar dimensions. As expected, both the resonant wavelength and the optical confinement factor in the active region increase sharply as the pillar size becomes larger (see Fig. 5(b) and (c)). This is mainly due to the buildup of effective refractive index that arises from the pillar size expansion. The evolution of the quality factor presented in Fig. 5(a) is more complicated. Similar to what has been observed in Fig. 4(e), when $w_{\text {act }}$ is fixed, the quality factor has minimal and maximal values around $L_{\text {act }}=300 \mathrm{~nm}$ and $L_{\text {act }}=650 \mathrm{~nm}$ respectively. However, when fixing $L_{\text {act }}$, a monotonic increase of quality factor is observed as $w_{\text {act }}$ is reduced. Generally speaking, longer pillar lengths help to lift up the optical mode, leading to less substrate leakage loss while narrower pillar widths help to minimize the mode distortion, resulting in radiative loss reduction. Quality factors close to $1 \times 10^{5}$ are obtained when $L_{\text {act }}=650 \mathrm{~nm}$ and $w_{\text {act }}=100 \mathrm{~nm}$, which is less than one order of magnitude worse than the original optimized $\mathrm{PhC}$ cavity without pillars.

The threshold gain calculated by performing a full analysis of the rate equations with carrier-dependent gain is presented in Fig. 5(d). As one can expect, the variation of $G_{\text {th }}$ as function of the pillar dimension is roughly opposite to the trend of the quality factor. To give a better view of the low threshold region, the inset of Fig. 5(d) shows $G_{\text {th }}$ as function of $w_{\text {act }}$ for $L_{\text {act }}$ fixed to $700 \mathrm{~nm}$. A minimal value of $10 \mathrm{~cm}^{-1}$ is obtained when $w_{\text {act }}=100 \mathrm{~nm}$. Since the confinement factor decreases sharply when $w_{\text {act }}$ drops below $100 \mathrm{~nm}, G_{\text {th }}$ increases again as $w_{\text {act }}$ becomes smaller than $100 \mathrm{~nm}$. The obtained very small threshold gain $\left(10 \mathrm{~cm}^{-1}\right)$ shows that the proposed laser cavity design is very promising for further experimental demonstration. Even in the case where a wider pillar width (200 nm, for example)
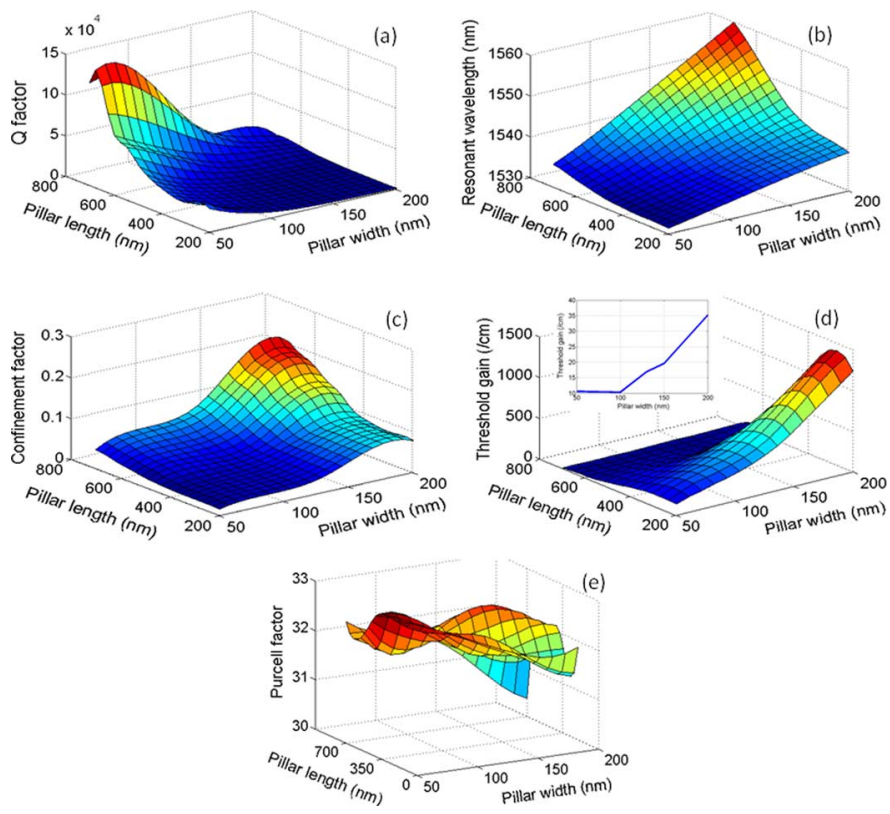

Fig. 5. Variation of (a) quality factor, (b) resonant wavelength, (c) optical confinement factor in active region, (d) threshold gain and (e) Purcell factor for different pillar width $\left(w_{\text {act }}\right)$ and pillar length $\left(L_{\text {act }}\right)$. Insert in $(d)$ : threshold gain calculated for different pillar width (wact) when Lact is fixed to be $700 \mathrm{~nm}$.

would be chosen for ease of fabrication, and fabrication errors introduce one order of magnitude higher loss, threshold gain can still be kept below $1000 \mathrm{~cm}^{-1}$, which can be easily provided by a InGaAs/InP heterostructure [30].

As predicted in Section II, the Purcell effect will be significant in this structure due to the compact mode volume. Since most of the parameters are fixed in (2), the calculated Purcell factor is mainly determined by the mode volume $\left(V_{\text {eff }} /(\lambda / n)^{3}\right)$. As already presented in Fig. 4(e), mode volume increases slightly as the pillar size increases, therefore the Purcell factor is maximized when the pillar size is small $(50 \mathrm{~nm} \times 200 \mathrm{~nm})$ while it is smaller when the pillar size is large $(200 \mathrm{~nm} \times 700 \mathrm{~nm})$. However, the calculated value does not change much across the whole parameter scan, and by using (3), the spontaneous emission factor is estimated to be larger than 0.96 in the worst case. Therefore, threshold-less laser behavior can be expected when other carrier loss mechanisms, e.g., surface non-radiative recombination, are negligible.

Due to the large surface-to-volume ratio of the $\mathrm{InGaAs} / \mathrm{InP}$ pillar, surface non-radiative recombination could become the critical factor limiting the laser performance though. Although it is widely recognized that InGaAs has at least one order of magnitude lower surface recombination velocity compared with other III-V materials, e.g., GaAs, there is still a large variation of measured values in literature, associated with the large differences in growth conditions and passivation treatments [34]-[36]. Here, we adopt the results from [34] as a starting point, and scan threshold pumping power as $v_{s}$ changes. The steady state solution of (6) is used in the calculation. A relative large pillar size $\left(w_{\text {act }}=150 \mathrm{~nm}, L_{\text {act }}=700 \mathrm{~nm}\right)$ is chosen in this case to relax the fabrication challenges. From the results presented in Fig. 6, it is found that although the threshold pump power is relatively high due to the larger active region size, 


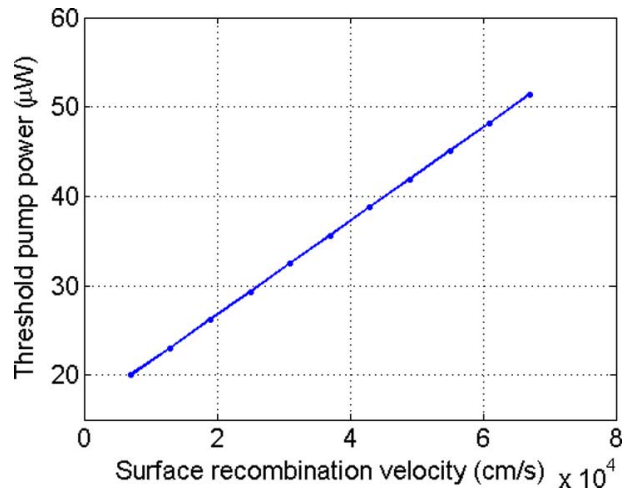

Fig. 6. Threshold pump power calculated for different surface recombination velocities. Parameters used for rate equation calculation are: $g 0=3000 \mathrm{~cm}^{-1}$, $B=0.7 \times 10^{-10} \mathrm{~cm}^{3} / \mathrm{s}, C=5 \times 10^{-29} \mathrm{~cm}^{6} / \mathrm{s}, N_{\mathrm{tr}}=1.1 \times 10^{18} \mathrm{~cm}-3$ and $N_{s}=5 \times 10^{18} \mathrm{~cm}^{-3}$, the pump light wavelength is $1.1 \mu \mathrm{m}$.

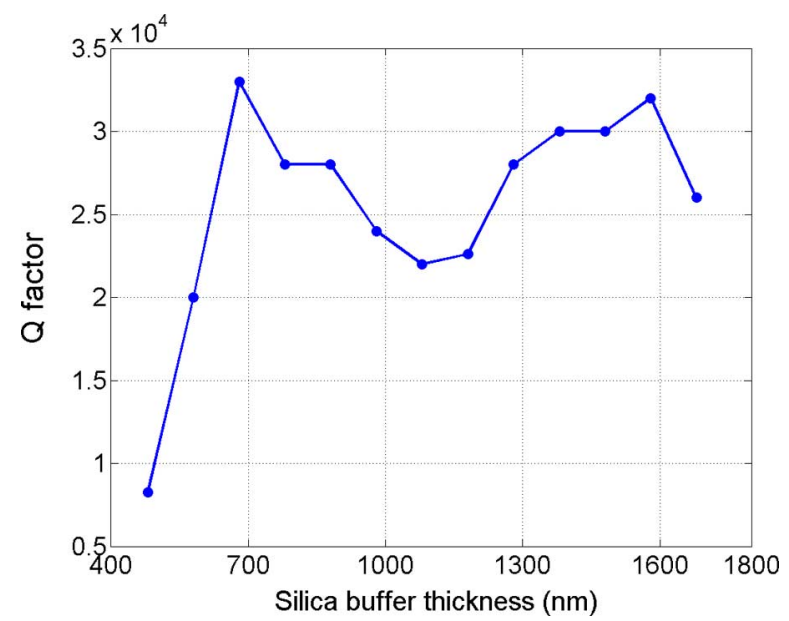

Fig. 7. Calculated quality factors for different silica buffer layer thicknesses. Pillar width and pillar length are $150 \mathrm{~nm}$ and $700 \mathrm{~nm}$, respectively.

it only increases by $100 \%$ when $v_{s}$ is increased by one order of magnitude. This proves that the proposed laser design can tolerate relatively high surface state density of the gain media.

The main fabrication challenge of this cavity design is the relatively high aspect ratio of the narrow trench that is etched down to the silicon substrate for the InGaAs/InP epitaxial growth. A thinner silica buffer is preferable to reduce the required etching depth. However, it will also result in higher leakage loss. In Fig. 7, we plot the calculated quality factor as a function of the silica buffer thickness. When the buffer is sufficiently thick, the quality factor varies mainly due to the interference between the downwards propagating light and the light reflected by the silica/silicon interface. Therefore, one can optimize the silica buffer thickness to obtain a higher quality factor. When the silica buffer is less than $600 \mathrm{~nm}$, leakage loss becomes dominant, decreasing the quality factor sharply. A thickness of $600 \mathrm{~nm}$ for the buffer layer seems to be a good trade-off between ease of fabrication and cavity performance.

\section{Thermal Dissipation of the Laser CAVity}

Heating considerably limits the performance of most $\mathrm{PhC}$ based micro-lasers and is mainly caused by the low conductivity of the typical cladding layers (air, silica) that prevents efficient
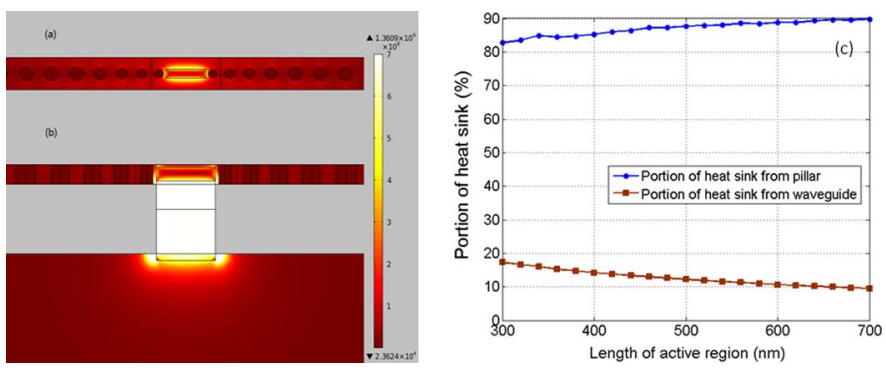

Fig. 8. (a) Top-view and (b) side-view of a typical heat flux distribution in the proposed laser cavity design $($ Lact $=700 \mathrm{~nm}$, wact $=150 \mathrm{~nm})$. (c) Calculated portion of heat that sinks from the InGaAs/InP pillar and the silicon waveguide.

heat dissipation. In the proposed laser configuration, since the InP pillar that connects the active region with the substrate has a high thermal conductivity $\left(68 \mathrm{Wm}^{-1} \mathrm{~K}^{-1}\right)$, it can act as a good heat sink. Moreover, by carefully choosing the pump wavelength, e.g., $1.3 \mu \mathrm{m}$, carriers will be generated only in the active region, eliminating other unwanted heating effects. In this section, we will analyze the thermal properties of the proposed laser design. All simulations are implemented in COMSOL, a commercial software package based on the finite-element-method (FEM). A volume source with energy density of $10^{16} \mathrm{~W} / \mathrm{m}^{2}$ is uniformly distributed across the active region. Only conductive heat transfer is simulated, while convection is neglected.

Fig. 8(a) and (b) show a typical heat flux distribution. It is obvious that the heat dissipation through the $\mathrm{InGaAs} / \mathrm{InP}$ pillar is indeed very efficient.

$$
P_{\text {pillar }}=\iint_{S_{\text {bottom }}} \varphi \cdot d S .
$$

In order to calculate the proportion of heat that is dissipated through the pillar, (8) is employed to integrate the heat flux that goes through the bottom surface. $\varphi$ is the calculated heat flux, and $S_{\text {bottom }}$ indicates the bottom surface of the pillar. Similarly, by replacing the integration surface with boundaries around the active region, one can also calculate the total heat flux. Simulation results can be found in Fig. 8(c). It can be found that most of the heat is conducted through the pillar even when the pillar size is small. More heat can be guided down to the substrate by increasing the pillar length $L_{\text {act }}$

$$
R_{\text {thermal }}=\frac{T_{\text {source }}-293.15[K]}{\iint_{S_{\text {total }}} \varphi \cdot d S} .
$$

Finally, (8) is employed to calculate the thermal resistance of the proposed laser design. $T_{\text {source }}$ is the average temperature in the active region, and the denominator of (8) is the total heat energy generated from the source. Simulation results are plotted in Fig. 9. As expected, the thermal resistance of laser cavities with a pillar is almost one order of magnitude smaller than that of cavities without pillars. Moreover, by increasing the pillar length, one can further decrease the thermal resistance. This is an important advantage of the proposed structure.

\section{CONCLUSION}

We have presented the design and detailed analysis of a micro-laser configuration that can be monolithically integrated on a silicon substrate. The laser operation mainly relies on 


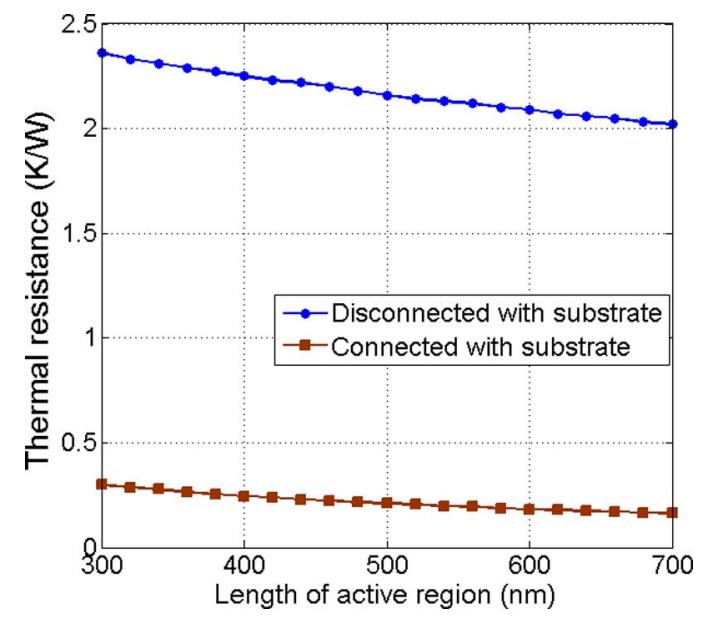

Fig. 9. Thermal resistance of the laser cavity calculated for different active region lengths, when there is a pillar or not between the active region and silicon substrate.

the close interaction between the silicon $\mathrm{PhC}$ cavity and the InGaAs/InP pillar inside. We have numerically proven that the increase of cavity loss after introducing the InGaAs/InP pillar is mainly substrate leakage rather than radiation loss, and it is possible to reduce it by optimizing the pillar dimensions. An overall quality factor as high as $1 \times 10^{5}$ has been achieved by employing a pillar size of $100 \mathrm{~nm} \times 650 \mathrm{~nm}$. We have also demonstrated the laser performance by detailed numerical calculations, and very low threshold operation $(\sim 10 \mu \mathrm{W})$ has been predicted even by taking surface non-radiative recombination into consideration. A remarkable Purcell effect in this cavity design has been observed, and a unity spontaneous emission factor is predicted. In addition, thermal dissipation analysis was performed and we have shown that most of the heat generated in the active region is guided through the InGaAs/InP pillar down to the silicon substrate, which greatly reduces the thermal resistance of the overall structure.

Compared with the work demonstrated in [14], [15] where similar III-V structures on silicon were grown for realizing high speed transistors, the main fabrication challenge of the proposed laser configuration lies in etching the relatively high aspectratio trench into the silicon substrate. A somewhat larger trench size (e.g., $150 \mathrm{~nm} \times 700 \mathrm{~nm}$ ) helps to relax the dry etching process, while still allowing for low threshold operation. Particular attention is still needed to obtain a vertical and smooth trench sidewall to prevent defect formation in the subsequent epitaxial growth. A protective mask, e.g., a thin silica layer, should be employed to ensure that the III-V materials only grow in the trench. Experimental verification of the different processes needed for realizing this device is currently being carried out. The proposed micro-laser configuration, which has ultra low-threshold and improved thermal dissipation, shows great promise for intra-chip optical communication and other applications that have stringent requirements on power consumption. It also shows the great potential and enormous benefits by bridging two unique material platforms - Silicon and III-V - in the epitaxy level, which may have significant impact on the photonics industry.

\section{REFERENCES}

[1] A. W. Fang, E. Lively, Y.-H. Kuo, D. Liang, and J. E. Bowers, "A distributed feedback silicon evanescent laser," Opt. Exp., vol. 16, pp. 4413-4419, Mar. 2008.

[2] L. Liu, R. Kumar, K. Huybrechts, T. Spuesens, G. Roelkens, E. Geluk T. Vries, P. Regreny, D. Thourhout, R. L. Baets, and G. Morthier, "An ultra-small, low-power, all-optical flip-flop memory on a silicon chip," Nat. Photon, vol. 4, pp. 182-187, Jan. 2010.

[3] M. Razeghi, M. Defour, R. Blondeau, F. Omnes, P. Maurel, O. Acher F. Brillouet, J. C. C-Fan, and J. Salerno, "First cw operation of a Ga0. 25In0.75As0.5P0.5-InP laser on a silicon substrate," Appl. Phys. Lett. vol. 53, pp. 2389-2391, Dec. 1988.

[4] M. Sugo, H. Mori, Y. Itoh, Y. Sakai, and M. Tachikawa, "1.5 $\mu \mathrm{m}$-Longwavelength multiple quantum well laser on a Si substrate," Jpn. J. Appl. Phys, vol. 30, pp. 3876-3878, Dec. 1991.

[5] S. Lourdudoss, "Heteroepitaxy and selective area heteroepitaxy for silicon photonics," Photon. Nanostr. Mater., Process. Characteriz., vol. 16, pp. 91-99, Apr. 2012.

[6] B. Kunert, S. Zinnkann, K. Volz, and W. Stolz, "Monolithic integration of $\mathrm{Ga}(\mathrm{NAsP}) /(\mathrm{BGa}) \mathrm{P}$ multi-quantum well structures on $\left(\begin{array}{lll}0 & 0 & 1\end{array}\right)$ silicon substrate by MOVPE," J. Cryst. Growth, vol. 310, pp. 4776-4779, Nov. 2008.

[7] S. Liebich, M. Zimprich, A. Beyer, C. Lange, D. Franzbach, and S. Chatterjee, "Laser operation onf Ga(NAsP) lattice-matched to (001) silicon substrate,” Appl. Phys. Lett., vol. 99, pp. 071109-071109, Aug. 2011.

[8] S. H. Huang, G. Balakrishnan, A. Khoshakhlagh, L. R. Dawson, and D L. Huffaker, "Simultaneous interfacial misfit array formation and antiphase domain suppression on miscut silicon substrates," Appl. Phys. Lett., vol. 93, pp. 071102-071102, Aug. 2008.

[9] E. Tournié, J. Reboul, L. Cerutti, J. Rodriguez, and P. Grech, “Continuous wave operation above room temperature of GaSb-based laser diodes grown on Si," Appl. Phys. Lett., vol. 99, pp. 121113-121113, Sep. 2011.

[10] Z. Wang, C. Junesand, W. Metaferia, C. Hu, L. Wosinski, and S. Lourdudoss, "III-Vs on Si for photonic applications-A monolithic approach,” Mater. Sci. Eng. B, vol. 177, pp. 1551-1557, Oct. 2012.

[11] J. Yang and P. Bhattacharya, "Integration of epitaxially-grown InGaAs/ GaAs quantum dot lasers with hydrogenated amorphous silicon waveguides on silicon," Opt. Exp., vol. 16, pp. 5136-5140, Mar. 2008.

[12] T. Wang, H. Liu, A. Lee, F. Pozzi, and A. Seeds, " $1.3-\mu \mathrm{m}$ InAs/GaAs quantum-dot lasers monolithically grown on Si substrates," Opt. Exp., vol. 19, pp. 11381-11386, Jun. 2011.

[13] T. A. Langdo, C. W. Leitz, M. T. Currie, E. A. Fitzgerald, A. Lochtefeld, and D. A. Antoniadis, "High quality Ge on Si by epitaxial necking," Appl. Phys. Lett., vol. 76, pp. 3700-3700, Jun. 2000.

[14] G. Wang, M. Leys, R. Loo, O. Richard, H. Bender, N. Waldron, G. Brammertz, J. Dekoster, W. Wang, M. Seefeldt, M. Caymax, and M. Heyns, "Selective area growth of high quality InP on Si (001) substrates," Appl. Phys. Lett., vol. 97, pp. 121913-121913, Sep. 2010.

[15] G. Wang, M. Leys, R. Loo, O. Richard, H. Bender, G. Brammertz, N. Waldron, W. Wang, J. Dekoster, M. Caymax, M. Seefeldt, and M. Heyns, "Selective area growth of InP and defect elimination on Si (001) substrates," J. Electrochem. Soc., vol. 158, pp. 645-650, Apr. 2011.

[16] J. S. Foresi, P. R. Villeneuve, J. Ferrera, E. R. Thoen, G. Steinmeyer, S. Fan, J. D. Joannopoulos, L. C. Kimerling, H. I. Smith, and E. P. Ippen, "Photonic-bandgap microcavities in optical waveguides," Nature, vol. 390, pp. 143-145, Nov. 1997.

[17] Y. Zhang, M. Khan, Y. Huang, J. Ryou, P. Deotare, R. Dupuis, and M. Lončar, "Photonic crystal nanobeam lasers," Appl. Phys. Lett., vol. 97, pp. 051104-051104, Aug. 2010.

[18] G. Shambat, B. Ellis, J. Petykiewicz, M. A. Mayer, and T. Sarmiento, "Nanobeam photonic crystal cavity light-emitting diodes," Appl. Phys. Lett., vol. 99, pp. 071105-071105, Aug. 2011.

[19] E. M. Purcell, "Spontaneous emission probabilities at radio frequencies," Phys. Rev., vol. 69, pp. 681-681, Jun. 1946.

[20] J. Huang, S. Kim, J. Gardner, P. Regreny, C. Seassal, P. Postigo, and A. Scherer, "Room temperature, continuous-wave coupled-cavity InAsP/InP photonic crystal laser with enhanced far-field emission directionality," Appl. Phys. Lett., vol. 99, pp. 091110-091110, Aug. 2011.

[21] H. Altug, D. Englund, and J. Vuckovic, "Ultrafast photonic crystal nanocavity laser,” Nat. Phys., vol. 2, pp. 484-488, Jul. 2006.

[22] T. Yoshie, M. Loncar, A. Scherer, and Y. Qiu, "High frequency oscillation in photonic crystal nanolasers," Appl. Phys. Lett., vol. 84, pp. 3543-3545, May 2004. 
[23] H. Y. Ryu, M. Notomi, E. Kuramoti, and T. Segawa, "Large spontaneous emission factor $(>0.1)$ in the photonic crystal monopole-mode laser," Appl. Phys. Lett., vol. 84, pp. 1067-1069, Feb. 2004.

[24] J. M. Gérard and B. Gayral, "Strong Purcell effect for InAs quantum boxes in three-dimensional solid-state microcavities," J. Lightw. Technol., vol. 17, pp. 2089-2095, Nov. 1999.

[25] T. Baba, "Photonic crystals and microdisk cavities based on GaInAsP-InP system," IEEE J. Sel. Top. Quantum Electron., vol. 3, pp. 808-830, Jun. 1997.

[26] Y. Zhang, M. Khan, Y. Huang, J. Ryou, P. Deotare, R. Dupuis, and M. LonCar, "Photonic crystal nanobeam lasers," Appl. Phys. Lett., vol. 97, pp. 051104-051104, Aug. 2010.

[27] A. Larrue, C. Wilhelm, G. Vest, S. Combrié, A. D. Rossi, and C. Soci, "Monolithic integration of III-V nanowire with photonic crystal microcavity for vertical light emission," Opt. Exp., vol. 20, pp. 7758-7770, Mar. 2012.

[28] B. Gunnar and Y. Yamamoto, "Analysis of semiconductor microcavity lasers using rate equations," IEEE J. Quantum Electron., vol. 11, pp. 2386-2396, Nov. 1991.

[29] T. F. Albrecht et al., "Transient four-wave mixing on (InGa) As/InP multiple quantum wells using a femtosecond optical parametric oscillator," Appl. Phys. Let., vol. 63, pp. 1945-1947, Oct. 1993.

[30] L. A. Coldren and S. W. Corzine, Diode Lasers and Photonic Integrated Circuits. New York: Wiley, 1995.

[31] P. B. Deotare and M. W. McCutcheon, "High quality factor photonic crystal nanobeam cavities," Appl. Phys. Lett., vol. 94, pp. 121106-121106, Mar. 2009.

[32] P. Velha, E. Picard, T. Charvolin, E. Hadji, J. C. Rodier, P. Lalanne, and D. Peyrade, "Ultra-high q/v fabry-perot microcavity on soi substrate," Opt. Exp., vol. 15, pp. 16090-16096, Nov. 2007.

[33] K. Srinivasan and O. Painter, "Momentum space design of high-Q photonic crystal optical cavities," Opt. Exp., vol. 10, pp. 670-684, Jul. 2002.

[34] M. Boroditsky, I. Gontijo, M. Jackson, R. Vrijen, E. Yablonovitch, T. Krauss, C.-C. Cheng, A. Scherer, R. Bhat, and M. Krames, "Surface recombination measurements on III-V candidate materials for nanostructure light-emitting diodes," J. Appl. Phys., vol. 87, pp. 3497-3497, Apr. 2000.

[35] E. Yablonovitch, R. Bhat, C. E. Zah, T. J. Gmitter, and M. A. Koza, "Nearly ideal InP/In0.53Ga0.47As heterojunction regrowth on chemically prepared In $0.53 \mathrm{Ga} 0.47 \mathrm{As}$ surfaces," Appl. Phys. Lett., vol. 60, pp. 371-373, Jan. 1992.

[36] G. Brammertz, M. Heyns, M. Meuris, M. Caymax, D. I. Jiang, Y. Mols, S. Degroote, M. Leys, and G. Borghs, "Surface recombination velocity in GaAs and In0.15Ga0.85As thin films," Appl. Phys. Lett., vol. 90, pp. 134102-134102, Mar. 2007.
Zhechao Wang received the B.S. degree in optical engineering and the Ph.D. degree in applied physics from Zhejiang University, China, and the Royal Institute of Technology, Sweden, in 2005 and 2010, respectively.

In 2011, he joined Ghent University as a postdoctoral scholar. He is engaged for the development of new fabrication processes for photonic devices, e.g., based on focused ion-beam etching, electron-beam lithography and epitaxial growth of III-Vs on silicon. He has published over 20 refereed papers in international journals. His research interests include silicon and III-V photonic integrated devices, micro and nano cavities, and epitaxial growth of semiconductor materials.

Bin Tian received the B.S. degree from Zhejiang University, China, and the M.S. degrees in optical engineering from the Royal Institute of Technology, Sweden.

He joined Ghent University as a Ph.D. candidate in 2011. His research interests mainly focus on silicon and III-V photonic integrated devices, epitaxial growth of III-Vs on silicon.

Dries Van Thourhout received the degree in physical engineering and the $\mathrm{Ph} . \mathrm{D}$. degree in applied physics from Ghent University, Ghent, Belgium, in 1995 and 2000, respectively.

From October 2000 to September 2002, he was with Lucent Technologies, Bell Laboratories, Murray Hill, NJ, where he was involved in the designing, processing, and characterization of $\mathrm{InP} / \mathrm{InGaAsP}$ monolithically integrated devices. In October 2002, he joined the Department of Information Technology, Ghent University, where he is currently a member of the permanent staff of the Photonics Group. He is a Lecturer or Co-Lecturer for four courses within the Ghent University Master in Photonics program (Microphotonics, Advanced Photonics Laboratory, Photonic Semiconductor Components and Technology). $\mathrm{He}$ is also coordinating the cleanroom activities of the research group. He is also engaged for the development of new fabrication processes for photonic devices, e.g., based on focused ion-beam etching and die-to-wafer bonding. He holds four patents. He has authored and coauthored more than 120 journal papers and invited papers at several major conferences. His research interests include the design, fabrication, and characterization of integrated photonic devices, silicon nanophotonic devices, and heterogeneous integration of InP-on-silicon. He holds an ERC-starting grant and received the 2012 "Laureaat van de Vlaamse Academie van Belgie" prize.

Dr. Van Thourhout is a member of IEEE Laser and Electrooptics Society and an Associate Editor for IEEE Photonics Technology Letters. 